\title{
Computational Studies on Effects of 1-(4-Chlorobenzylideneamino)-4-Phenyl-1H- Imidazol-2-Amine as an Anticancer Drug on DNA
}

\author{
Soroor Soraya and Seyed Sina Soraya
}

\begin{abstract}
Extensive studies conducted by investigators have led into discover of anticancer properties of compounds drived from Imidazole. Considering the fact that the series of compounds being new and the defects in the drugs available, which cause complications in the DNA string, the need for finding ways to cope that challenge is immiment. The value and the accuracy of computational methods, led us to choose the 4 nucleotides, Adenine, Cytosine, Guanine and Thymine as a measure of the DNA string and study the effect of the compound 1-(4-chlorobenzylideneamino)-4-phenyl-1H-imidazol-2-amine as a new biologic Imidazole anticancer drug, the medicinal effect of which has recently been established, on any of these DNA string nucleotides. The studies reveal the fact that the highest amount of interaction between the drug and the nucleotide is on Guanine. Then to conduct a through careful review, we checked all different constructible possibilities of drug containing (Imidazole ring, Phenyl and Chlorine atom) and Guanine nucleobase.
\end{abstract}

IndexTerms-1-(4-chlorobenzylideneamino)-4-phenyl-1Himidazol-2-amine, nucleotide, nucleobase, anticancer drug.

\section{INTRODUCTION}

Extensive studies conducted by investigators have been led to the discovery of anticancer properties of some compounds [1]. Considering the fact that the series of Imidazol compounds are brand new [2] and the defects in the drugs available, results in defragmentations and breaks in the DNA string [3], the need for finding ways to cope with that problem felt more than ever, on the other hand, because the string of DNA containing the nucleotide sequence is repeated sequentially [4], it is logical to limit the study to this strands and making the calculations simpler. Considering these points in this study, four nucleotides (Adenine, Cytosine, Guanine and Thymine) examined by using accurate Quantum Mechanical methods and the base optimum structure of these nucleotides investigated under the influence of the drug.

2-Amino-1-arylidenaminoimidazoles, a novel class of orally (po) active microtubule-destabilizing anticancer agents, were synthesized. The compounds were designed from a hit compound identified in a drug discovery platform by using

Manuscript received March 1, 2012; revised March 31, 2012

Soroor Soraya is with the Basic Science Faculty, Department of Chemistry, Islamic Azad University, Neyriz Branch, Fars, Iran (corresponding author to provide phone: +987116274818; fax: +987116267974; e-mail: soroor_soraya@yahoo.com).

Seyed Sina Soraya is with the Department of IT and Computer Engineering, Shiraz University, Shiraz, Iran (e-mail: sorayya-sina@att.com). cancer cell-based high throughput screening assay. Selective synthesized compounds exerted cell cytotoxicity against human cancerous cells. These new po active antimitotic anticancer agents are to be further examined in preclinical studies and developed for clinical uses [2]. Scheme 1 shows the general method for the synthesis of 2-amino-1-arylidenaminoimidazoles. Starting with aminoguanidine, the free amine was obtained by adding hydrochloride to liberate carbon dioxide, coupled with 4-chlorobenzaldehyde to generate guanylhydrazone (1) in a good yield of $91 \%$. Ring closure reaction was accomplished by the treatment of 2-bromoacetophenone with 1 to afford 1-(4-chlorobenzylideneamino)-4-phenyl- $1 H$-imidazol-2-ami ne (2) in a yield of $77 \%$ [5]-[8].

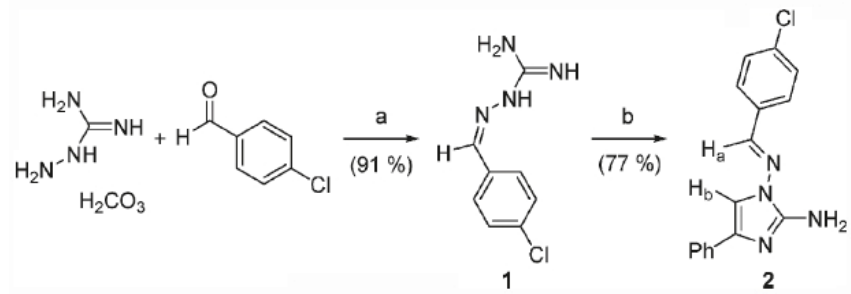

Sheme1. Reagents and conditions:

(a) 4-chlorobenzaldehyde, reflux, $10 \mathrm{~min}$;

(b) 2-bromoacetophenone, $\mathrm{NaOH}, \mathrm{EtOH}, 70 \_\mathrm{C}, 4 \mathrm{~h}$.

Among the derivatives of

2-Amino-1-arylidenaminoimidazoles,

$1-(4-$ chlorobenzylideneamino)-4-phenyl- $1 H$-imidazol-2-amine chosen regarding its crucial role in the cytotoxic activity [2].

Simulation calculations have not been done on this compound but several calculations have been performed on similar various compounds. Apparently performing a lab research, demanding purchase of material in addition to having sufficient time and equipped lab conversely a job performed by a simulation method is an approach which transfers a computer to a virtual lab therefore we can conclude simulation can be conclude as a lab experience.

In this study, we intend to perform high accuracy Quantum Mechanical methods to reach the biological effects of Imidazole new anticancer drug which in terms used for treating related diseases.

\section{METHODS}

Methods of calculations, are based on theory and also electron-molecular calculations which under taken by 
Gaussian $^{\mathrm{TM}}$ program. The research performed in such way that the stable structures of DNA nucleotides and drug, using density functional theory, B3LYP method and LANL2DZ basis set; separately optimized. There after we investigate the combination of drug and nucleotide with Quantum Chemical methods. To ensure that the structures which have been analyzed are at their minimum energy level, the frequency calculations also performed in addition to the former methods Using the natural bond orbital analysis, we calculated each atomic charge and the rate of exchanges in charges, happens due to the interaction between each nucleotides and drug which in terms brought into consideration. Considering all the possibilities related to the interaction between DNA and constructive nucleotides of the DNA string, the most probable conditions are proposed. The energy produced from the interaction of drug and nucleotides calculated with the following formula.

$$
E_{\text {int. }}\left(\mathrm{kJ} . \mathrm{mol}^{-1}\right)=2625.50\left[E_{D N A-d r u g}(\mathrm{au})-E_{D N A}(\mathrm{au})-E_{\text {drug }}(\mathrm{au})\right](1)
$$

\section{A. Gaussian 03}

Gaussian has been designed with the needs of the user in mind. All of the standard input is free-format and mnemonic. Reasonable defaults for input data have been provided, and the output is intended to be self-explanatory. Mechanisms are available for the sophisticated user to override defaults or interface their own code to the Gaussian system. The authors hope that their efforts will allow users to concentrate their energies on the application of the methods to chemical problems and to the development of new methods, rather than on the mechanics of performing the calculations [9].

\section{B. B3LYP Method}

The popular B3LYP (Becke, three-parameter, Lee-Yang-Parr) [10] is one of the hybrid functionals that exchange-correlation functional is:

$$
E_{x c}^{B 3 L Y P}=\left(E_{x c}^{L D A}+a_{0}\left(E_{x}^{H F}+E_{x}^{L D A}\right)+a_{x}\left(E_{x}^{G G A}-E_{x}^{L D A}\right)+a_{c}\left(E_{c}^{G G A}-E_{c}^{L D A}\right)\right.
$$

where $a_{0}=0.20, a_{x}=0.72$ and $a_{c}=0.81$ are the three empirical parameters determined by fitting the predicted values to a set of atomization energies, ionization potentials, proton affinities, and total atomic energies [11]; $E_{x}^{G G A}$ and $E_{c}^{G G A}$ are generalized gradient approximations: the Becke 88 exchange functional [12] and the correlation functional of Lee, Yang and Parr [13], and $E_{c}^{L D A}$ is the VWN local-density approximation to the correlation functional [14].

\section{LANL2DZ Basis Set}

Most methods require a basis set be specified; if no basis set keyword is included in the route section, then the STO-3G basis will be used. The exceptions consist of a few methods for which the basis set is defined as an integral part of the method [15]; It is a subclass of Effective Core Potential (ECP) Basis sets that Los Alamos National Laboratories (LANL), 2 is the version number and $\mathrm{DZ}$ indicates that it is a "double-zeta" basis set [16].

\section{RESULTS AND DisCUSSIONS}

Detailed studies on the structure of each of the nucleotides (Adenine, Cytosine, Guanine and Thymine) and Guanine nucleobase, which are proven to be the fundaments of DNA string, achieved by using method of density functional theory (DFT), (B3LYP) method and (LANL2DZ) basis set. Fig. 1 to 4 show the optimized structures for nucleotides (Adenine, Cytosine, Guanine and Thymine).

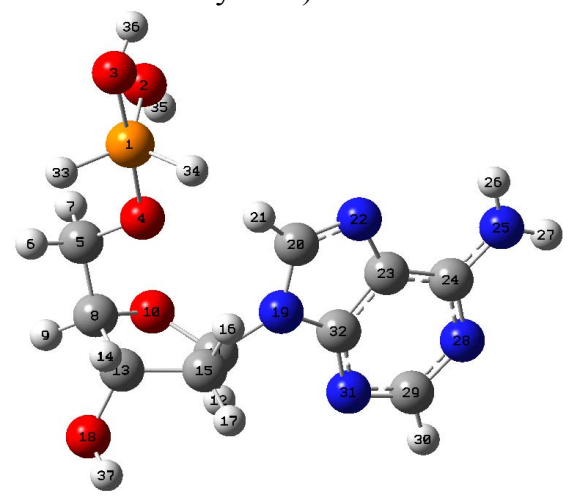

Fig. 1. The optimum structure of Adenine by using B3LYP/LANL2DZ (Illustrated as Nitrogen atom in blue, red representing Oxygen, orange; Phosphorus, gray; Carbon and white; Hydrogen respectably.)

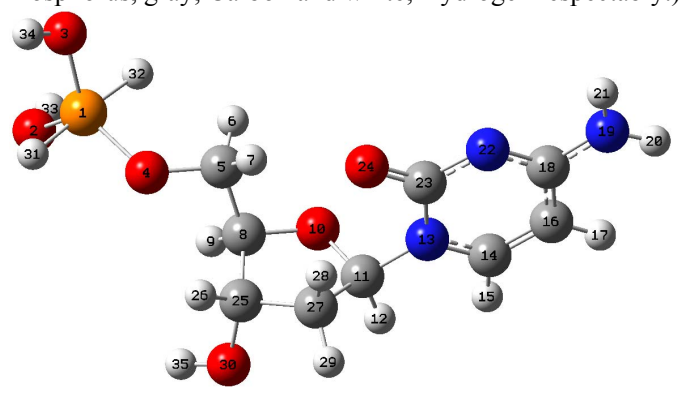

Fig. 2. The optimum structure of Cytosine by using B3LYP/LANL2DZ. (Illustrated as Nitrogen atoms in blue, red representing Oxygen, orange; Phosphorus, gray; Carbon and white for Hydrogen respectably.)

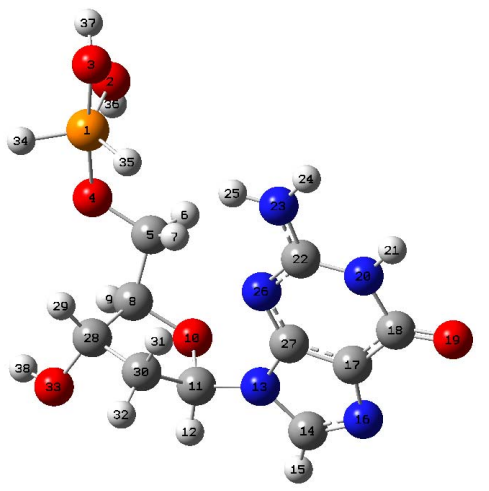

Fig. 3. The optimum structure of Guanine achieved by using B3LYP/LANL2DZ. (Illustrated as Nitrogen atoms in blue, red; Oxygen, orange; Phosphorus, gray; Carbon and white for Hydrogen respectably.)

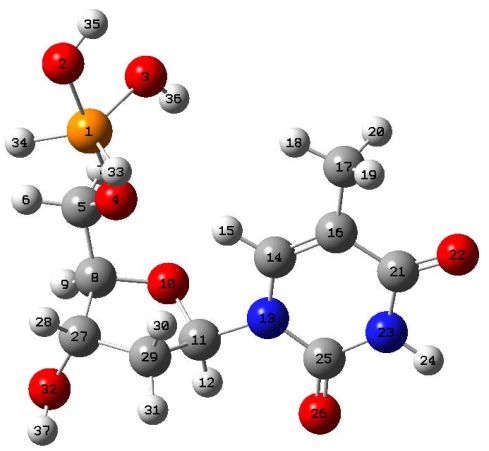

Fig. 4. The optimum structure of Thymine by using B3LYP/LANL2DZ. (Illustrated as Nitrogen atoms in blue, red; Oxygen, orange; Phosphorus, gray; Carbon and white for Hydrogen respectably.) 
Also we we decided to optimize Guanine nucleobase. Fig. 5 show the optimized structures for Guanine nucleobase.

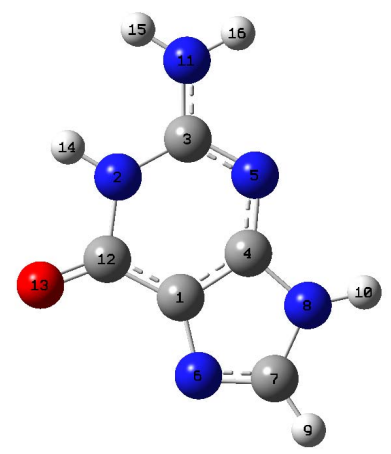

Fig. 5. The optimum structure of Guanine nucleobase by using B3LYP/LANL2DZ. (Illustrated as Nitrogen atoms in blue, red; Oxygen, gray; Carbon and white for Hydrogen respectably.)

In all of the mentioned structures in addition to the nucleobases, Tetrahydrofuran hetrocycle, chain which contains Phosphorus, Oxygen is also integrated .The special properties of these systems are due to the existing . electrons and electro negativity atoms which are capable of producing hydrogen binding interactions as a non-Covalent bond. Hydrogen bond is responsible for making living macromolecular systems such as two chains of DNA and protein and also complex structures. In live and artificial systems designed for having accurate and regular donor and acceptor sites of hydrogen bond in structural components is very critical and important. This is the result of one-dimensional chains of hydrogen bonds $\mathrm{N}-\mathrm{H} . . \mathrm{O}=\mathrm{C}$ and regular structure in the solid state [17].

These observations considered to provide basis for controlling live structures in order to have an attempt to improve the effectiveness of drugs through their rings and the groups.

In the second part, we optimized the drugs structure generally used to target the cancerous cells for curing process in cancers by indicating topoisomerase [18] level of activity so we would be able to aim the regarded DNA nucleotides.

For analyzing a stable molecular structure we investigated molecules having desired structure by torsion in bonds N_21-H_33 and C_8-H_26 (Fig. 6) As a result it is observed that the structure level of energy for the regarded compound is not stable and considering this fact all structural calculations performed with the lowest possible energy level for achieving a stable structure (with a energy difference of $27.89 \mathrm{~kJ} \cdot \mathrm{mol}^{-1}$ ).

Fig. 7 shows the stable optimized structure of the drug appears in theoretical level of B3LYP/LANL2DZ.

Fig. 8 shows the most stable and optimized structure of the drug and the relative charges on each atom.

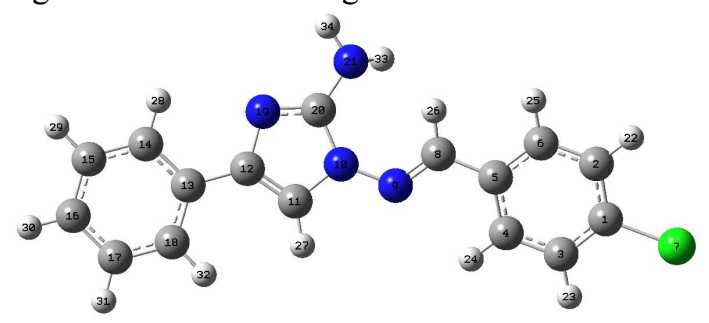

Fig. 6. The optimum structure of the drug achieved by using B3LYP/LANL2DZ with torsion in bonds N_21-H_33 and C_8-H_26. (Illustrated as Nitrogen atoms in blue, green; Chlorine, gray; $\bar{C}$ arbon and white for Hydrogen respectably.)

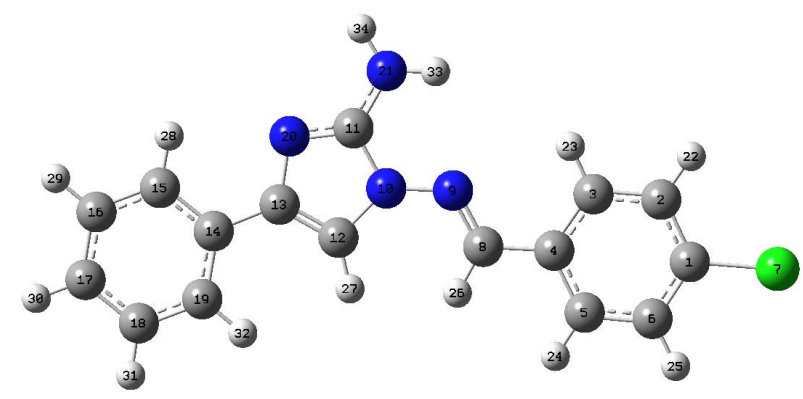

Fig. 7. The optimum structure of the most stable form relating to drug by using B3LYP/LANL2DZ. (Illustrated as Nitrogen atoms in blue, green;

Chlorine, gray; Carbon and white for Hydrogen respectably.)

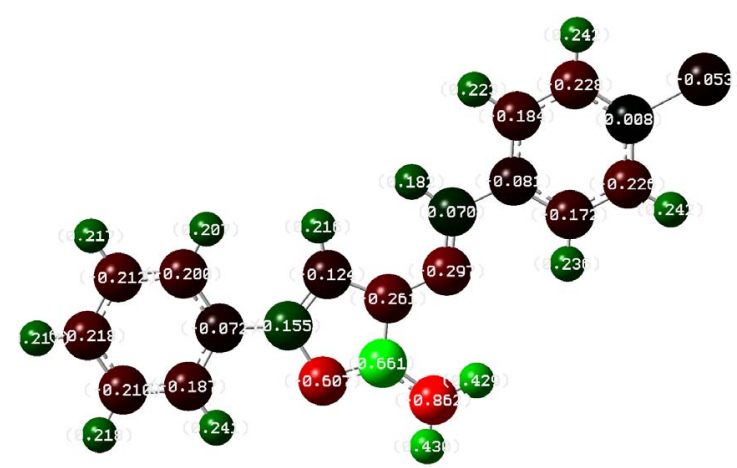

Fig. 8. The drug structure and relative charge on each of its atoms (darker color indicates a negative charges and bright colors show positive charges.)

The third part of this study which presents the main part of this article aims to find the stable structure of nucleotide-drug complexes. To find a stable structure of these complexes, the different probabilities were used after finding stable molecular structures of nucleotides and drug using density functional theory with complete basis functions. Structure and composition of each of the nucleotides and Imidazole compound shows that the drug position is in the distance of 3 $\AA$ from the nucleotid in order to make stable complexes (Fig. 9).

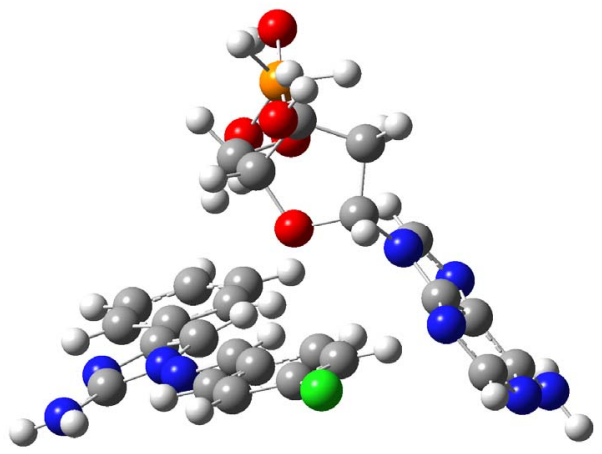

(a)

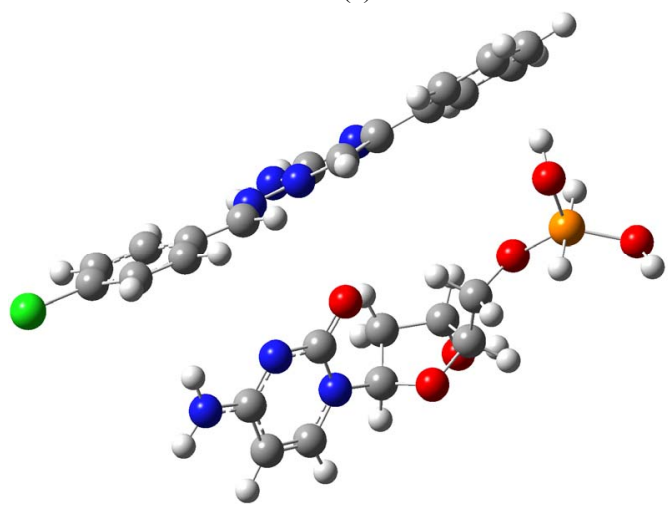

(b) 


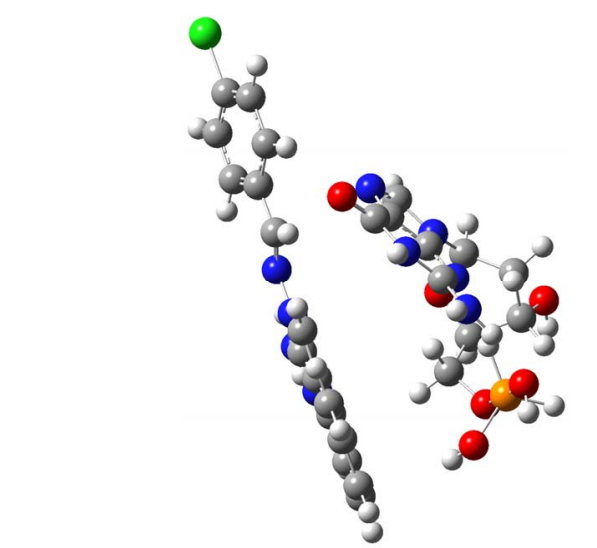

(c)

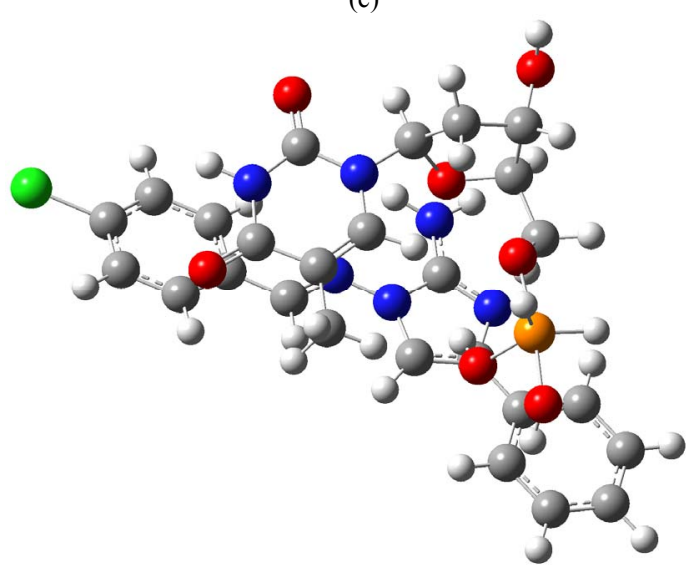

(d)

Fig. 9. The optimum structure of each system which surveyed. (a). Adenine nucleotide drug complex; (b). Cytosine nucleotide_drug complex; (c). Guanine nucleotide_drug complex; (d). Thymine nucleotide_drug complex. (Illustrated as Nitrogen atoms in blue, red; Oxygen, green; Chlorine, orange; Phosphorus, gray; Carbon and white for Hydrogen respectably.)

In the forth step the complex structure of the Guanine nucleobase-drug having the stable Guanine nucleotide structure was checked for comparison. To conduct a through careful review it was necessary to check all different constructible possibilities after finding a stable nucleobase molecular structures and drug which are studied during the survey . For structural studies on three different positions of drug containing Imidazole ring, Phenyl and Chlorine atom were conducted to determine that Guanine nucleobase initiates reaction with which possible active site of the drug.

Each of the mentioned pictures that show in fig. 9 illustrate the fact that in all cases after the optimization process the highest tendency of cross reaction for nucleobases of Guanine is with the terminal site in the drugs structure namely the Phenyl.

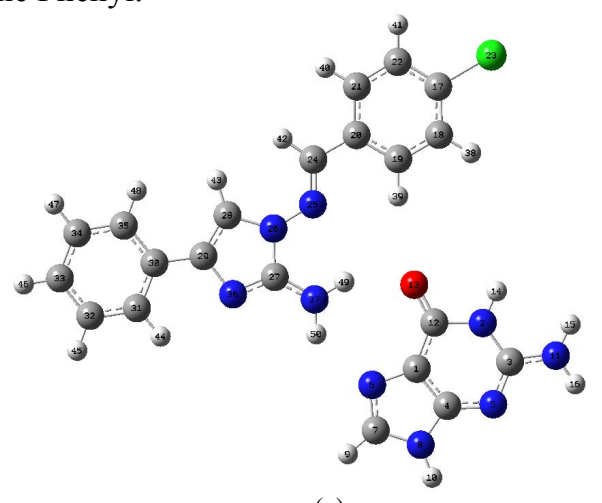

(a)

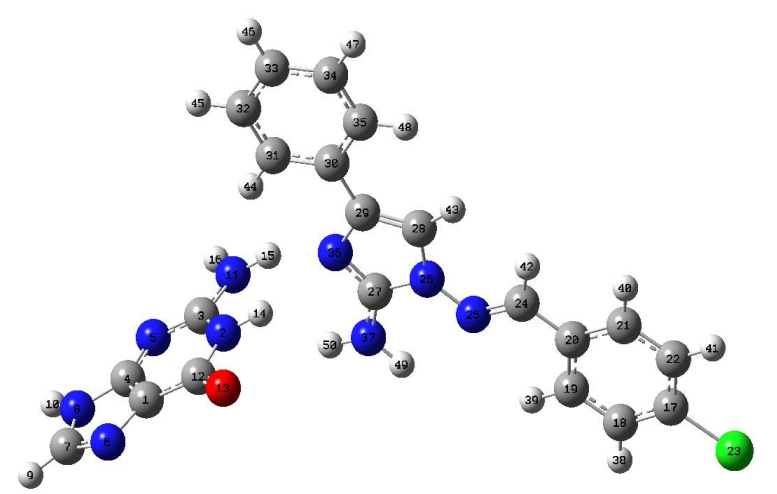

(b)

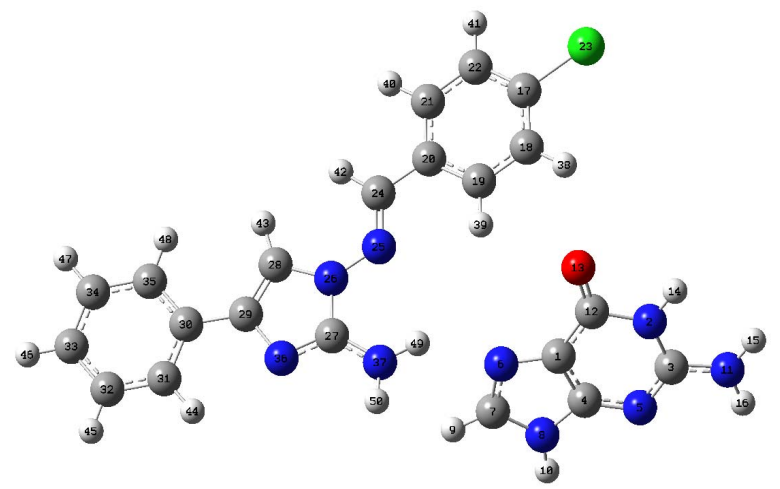

(c)

Fig. 10. The optimum structure of each system that studied. (a). Initial position of Guanine nucleobase_drug complex was located near Imidazole ring; (b). Initial position of Guanine nucleobase_drug complex was located near Phenyl; (c). Initial position of Guanine nucleobase_drug complex was located near Chlorine atom. (related to the Nitrogen atom in blue, red;

Oxygen, green; Chlorine, gray; Carbon and white; Hydrogen.)

\section{CONCLUSION}

The energies related to the interaction of drug and each nucleotide were calculated by single-Point B3LYP/LANL2DZ calculations which were ranged from -21.2 $\mathrm{kJ} / \mathrm{mol}$ (Thymine-drug) to $-49.63 \mathrm{~kJ} / \mathrm{mol}$ (Guanine-drug). The interaction energy recorded for Adenine is $-36.94 \mathrm{~kJ} / \mathrm{mol}$ and for Cytosine is $-25.1 \mathrm{~kJ} / \mathrm{mol}$ (table. I). Regarding the fact that amount of energies are close to the experimental values that is an indicator for the connection of the drug and nucleotides of DNA, it can be proposed to increase the effectiveness of drug in healing process ,capsules must be designed to deliver the drug to the targeted nucleotide which should have less interaction energy than high energy amounts. Moreover, in recent cases the distance between hydrogen atoms and drug electronegativity atoms of drug is less than 2 angstrom that indicates the hydrogen bonds between the molecular structure of drug and DNA.

In the other hand because of the most interaction energy is between drug and Guanine Nucleotide, we decided to check interaction energies between Guanine nucleobase and drug on the different positions of drug. About Guanine nucleobase-drug complex that after optimizing, the highest tendency of reaction for nucleobases of Guanine is with the Phenyl. The interaction energy for this complex by single-point B3LYP/LANL2DZ is $-57.25(\mathrm{~kJ} / \mathrm{mol})$.

Table. II shows interaction energies for structural studies on three different positions of drug containing Imidazole ring, 
Phenyl and Chlorine atom.

TABLE I: INTERACTION ENERGY VALUES BETWEEN DRUG AND EACH NUCLEOTIDE OF DNA (KJ/MOL).

\begin{tabular}{|c|c|c|c|}
\hline Adenine & Thymine & Cytosine & Guanine \\
\hline-36.94 & -21.2 & -25.10 & -49.63 \\
\hline
\end{tabular}

TABLE II: INTERACTION ENERGY VALUES BETWEEN DRUG AND GUANINE NUCLEOBASE (KJ/MOL).

\begin{tabular}{|c|c|}
\hline Imidazole ring & -48.74 \\
\hline Phenyl & -57.25 \\
\hline Chlorine atom & -38.20 \\
\hline
\end{tabular}

Fist row, the initial position of Guanine nucleobase-drug copmlex is near Imidazole ring. Second row, the initial position of Guanine nucleobase-drug complex is near Phenyl. Third row, the initial position of Guanine nucleobase-drug complex is near Chlorine atom.

\section{REFERENCES}

[1] G. W. Gribble, The alkaloids, Academic Press, 1990, New York

[2] W. T. Li, D. R. Hwang, J. S. Song, C. P. Chen, J. J. Chuu, C. B. Hu, H. L. Lin, C. L. Huang, C. Y. Huang, H. Y. Tseng, C. C. Lin, T. W. Chen, C. H. Lin, H. S. Wang, C. C. Shen, C. M. Chang, Y. S. Chao, and C. T. Chen, "Synthesis and biological activities of 2-Amino-1-arylidenamino Imidazoles as orally active anticancer agents," J. Med. Chem, vol. 53, pp. 2409-2417, 2010.

[3] P. Deepa, P. Kolandaivel, and K. Senthilkumar, "Interaction of Anticancer drugs with usual and mismatch base pairs-density functional theory studies," Biophys. Chem, vol. 136, pp. 50-58, 2008.

[4] J. M. Butler, Forensic DNA typing, elsevier, 2001, pp. 14-15.

[5] M. Z. Krimer, F. Z. Makaev, E. P. Styngach, A. G. Koretskii, S. I. Pogrebnoi, and A. I. Kochug, "Synthesis of substituted 2-amino-1-arylidenaminoimidazoles and 1-arylidenaminoimidazo[1,2-a]imidazoles," Chem. Heterocycl. Compd., vol. 32, pp. 1035-1039, 1996.
[6] N. N. Kolos, V. D. Orlov, B. V. Paponov, and O. V. Shishkin "synthesis of dihydro derivatives of 2-amino-4,5,7-triarylimidazo-[1,5-b]pyridazine," Chem. Heterocycl. Compd., vol. 35, pp. 1207-1213, 1999.

[7] L. Quattara, M. Debaert, and R. Cavier. "Synthese et active antiparasitaire de nouveaux derives du nitro-5 imidazol," Farmaco, vol. 42, pp. 449-456, 1987.

[8] A. V. Ivashchenko, V. T. Lazareva, E. K. Prudnikova, S. P. Ivashchenko, and V. G. Rumyantsev, "Synthesis of 1,2-diaminoimidazole guanylhydrazone with $\alpha$-haloalkyl aryl ketones," Chem. Hetrocycl. Compd., vol. 18, pp. 185-189, 1982.

[9] Gaussian 03 program; Help table of contents;Preparing input files;Basis sets.

[10] P. J. Stephens, F. J. Devlin, C. F. Chabalowski, and M. J. Frisch. "Ab initio calculation of vibrational absorption and circular dichroism spectra using density functional force fields," J. Phys. Chem., vol. 98, no. 45 , pp. $11623-11627,1994$.

[11] A. D. Becke, "Density-functional thermochemistry. III. The role of exact exchange," J. Chem. Phys., 1993, vol. 98, no. 7, pp. 5648-5652.

[12] A. D. Becke, "Density-functional exchange-energy approximation with correct asymptotic behavior," Phys. Rev, vol. A 38, no. 6, pp. 3098-3100, 1988

[13] C. Lee, W. Yang, and R. G. Parr, "Development of the colle-salvetti correlation-energy formula into a functional of the electron density," Phys. Rev., vol. B 37, no. 2, pp. 785-789, 1988.

[14] S. H. Vosko, L. Wilk, and M. Nusair, "Accurate spin-dependent electron liquid correlation energies for local spin density calculations: a critical analysis," Can. J. Phys., vol. 58, no. 8, pp. 1200-1211, 1980.

[15] Gaussian 09 user's reference. [Online]. Available: $\mathrm{http}: / / \mathrm{www}$.gaussian.com $/ \mathrm{g}$ tech $/ \mathrm{g}$ ur $/ \mathrm{m}$ basis sets.htm.

[16] M. B. Roman. "Intramolecular basis set superposition error as a measure of basis set incompleteness: Can one reach the basis set limit without extrapolation?" J. Chem. Phys., vol. 132, pp.150-154, 2010.

[17] Y. Haketa, N. Eifuku, Y. Bando, I. Yamada, A. Hagihara, and H. Maeda, "Solid-state hydrogen-bonding self-assemblies and keto-enol tautomerism of 1,3-dipyrrolyl-1,3-propanediones; Supramolecular chemistry,", vol. 23, pp.209-217, 2011.

[18] D. L. Ma, D. Shiu-Hin Chan, P. Lee, M. Hiu-Tung Kwan, and C. H. Leung, "Molecular modeling of drug-DNAinteractions: Virtualscreening to structure-baseddesign," vol. 93, pp. 1252-1266, 2011. 